\title{
Geißblatt-Arten auf Sachalin, Russland
}

\author{
Victor V. Sheiko \& Gennady Firsov
}

\begin{abstract}
On the Sakhalin island, six species of honeysuckles are native: Lonicera caerulea, L. tolmatchevii, L. glehnii, L. maximowiczii subsp. sachalinensis, L. chamissoi und L. chrysantha. These very attractive and robust species are described. They could be used as ornamental plants also for gardens in middle Europe.

\section{Zusammenfassung}

Auf der Insel Sachalin gibt es sechs heimische Geißblatt-Arten: Lonicera caerulea, L. tolmatchevii, L. glehnii, L. maximowiczii subsp. sachalinensis, L. chamissoi und L. chrysantha. Diese werden hier vorgestellt. Da sie sehr attraktiv und meist auch robust sind, eignen sie sich als Zierpflanzen auch für Gärten in Mitteleuropa.
\end{abstract}

\section{Klimatische und geografische Verhältnisse auf Sachalin}

Die Insel Sachalin liegt nördlich von Japan und wurde vor etwa 7000 Jahren vom Festland getrennt. Das Klima ist rau. Östlich von Sachalin ist das Meer sehr kalt und in der zweiten Winterhälfte mit Eis bedeckt. Westlich wird Sachalin durch das Japanische Meer begrenzt. Die Meerenge ist hier an ihrer schmalsten Stelle nur $6 \mathrm{~km}$ breit. Durch sie gelangt von Süden her der warme Tsusima-Strom, so dass das westliche Sachalin klimatisch deutlich begünstigter ist als der Osten. Zwischen Sachalin und der benachbarten japanischen Insel Hokkaido verläuft die $40 \mathrm{~km}$ breite Laperuz-Straße.

Sachalin ist durch zwei bis $1500 \mathrm{~m}$ hohe Gebirge gekennzeichnet, die sich vom Norden in den Süden erstrecken. Diese sind voneinander durch breite, sumpfige Täler getrennt: das Tym-Poronayskaya-Tal in der Inselmitte und das Susunayskaya-Tal im Süden. Im letzteren befindet sich der botanische Garten von Sachalin, der von der fernöstlichen Außenstelle der Russischen Akademie der Wissenschaften verwaltet wird. Der Garten ist ein Zentrum zur Erforschung und zum Schutz der lokalen Flora.

Der Sommer auf Sachalin ist kühl und gewöhnlich in seiner zweiten Hälfte regenreich. Im Winter gibt es viel Schnee. Die tiefsten Temperaturen liegen im Süden bei $-30^{\circ} \mathrm{C}$ und im Norden noch $10^{\circ} \mathrm{C}$ darunter. Der Frühling beginnt erst etwa im April/Mai und dauert lange, wobei es zu täglichen starken Temperaturschwankungen kommen kann. Der Herbst ist wärmer als in den benachbarten Gebieten des kontinentalen Russlands.

\section{Kurzer Einblick in Vegetation und Flora} Ein großer Teil der Insel ist mit dunklen Nadelwäldern aus Picea ajanensis und Abies sachalinensis bedeckt. Im Norden treten Lärchenwälder (mit Larix cajanderi) auf. An den Flüssen findet man Weiden-Pappel-Wälder sowie Bestände aus Breitblättriger Birke (Betula platyphylla). Im Süden gibt es Mischwälder aus Nadel- und Laubbäumen.

Ab einer Höhe von 600 m werden die dunklen Nadelwälder durch lichtere Birkenwälder ersetzt, in denen Betula ermannii, Bambus (verschiedene Sasa-Arten) und Sträucher wie Acer ukurunduense, Euonymus sachalinensis und Prunus nipponica vorkommen. In Höhenlagen um $800 \mathrm{~m}$ beginnt die Krummholzzone mit krüppelig wachsender Betula ermannii und einem Dickicht aus Pinus pumila. Darüber gedeiht ein Gürtel aus Tundravegetation. Der Norden Sachalins trägt eine circumboreale Vegetation, während die Pflanzendecke im Süden Sachalins ostasiatisch beeinflusst ist. Im Süden gedeihen Koniferen und immergrüne Sträucher wie Skimmia und Ilex.

Als Besonderheit ist zu nennen, dass Sachalin während der Eiszeit nicht von Gletschern bedeckt war. So konnten sich einige Arten aus der voreiszeitlichen Flora halten. Zu den besonders interessanten Pflanzen Sachalins gehören u. a. sechs laubwerfende Geißblatt-Arten, die auf Sachalin wild vorkommen und im Folgenden genauer vorgestellt werden sollen. Innerhalb der 
Gattung Lonicera gehören sie zu verschiedenen Gruppen.

\section{Lonicera caerulea $\mathrm{L}$.}

Der Strauch wird $2 \mathrm{~m}$ hoch. Charakteristisch sind seine Zweige mit der braunen, abblätternden Borke. In den Blütenständen sind stets zwei Blüten vereinigt. Die Blütenkrone ist meist 9-15 mm lang, fast radiärsymmetrisch und hellgelb bis grünlichgelb gefärbt. Die Beeren sind rundlich bis eiförmig-elliptisch und werden 7-12 mm lang. Die Bezeichnung caerulea bezieht sich auf ihre blau bereifte Färbung. Viele Lonicera-Arten sind bekanntlich giftig. Lonicera caerulea ist jedoch ein guter Fruchtstrauch, der wegen seiner früh reifenden Beeren häufig kultiviert wird. Seine Früchte schmecken süß-sauer. Bitter schmeckende Formen, die in Europa und West-Sibirien gepflanzt werden, fehlen auf Sachalin. Lonicera caerulea wird außerdem als Zierpflanze und Bienenweide kultiviert.

Auf Sachalin kommt $L$. caerulea vor allem im Norden und in der Mitte vor. Sie tritt in ver-

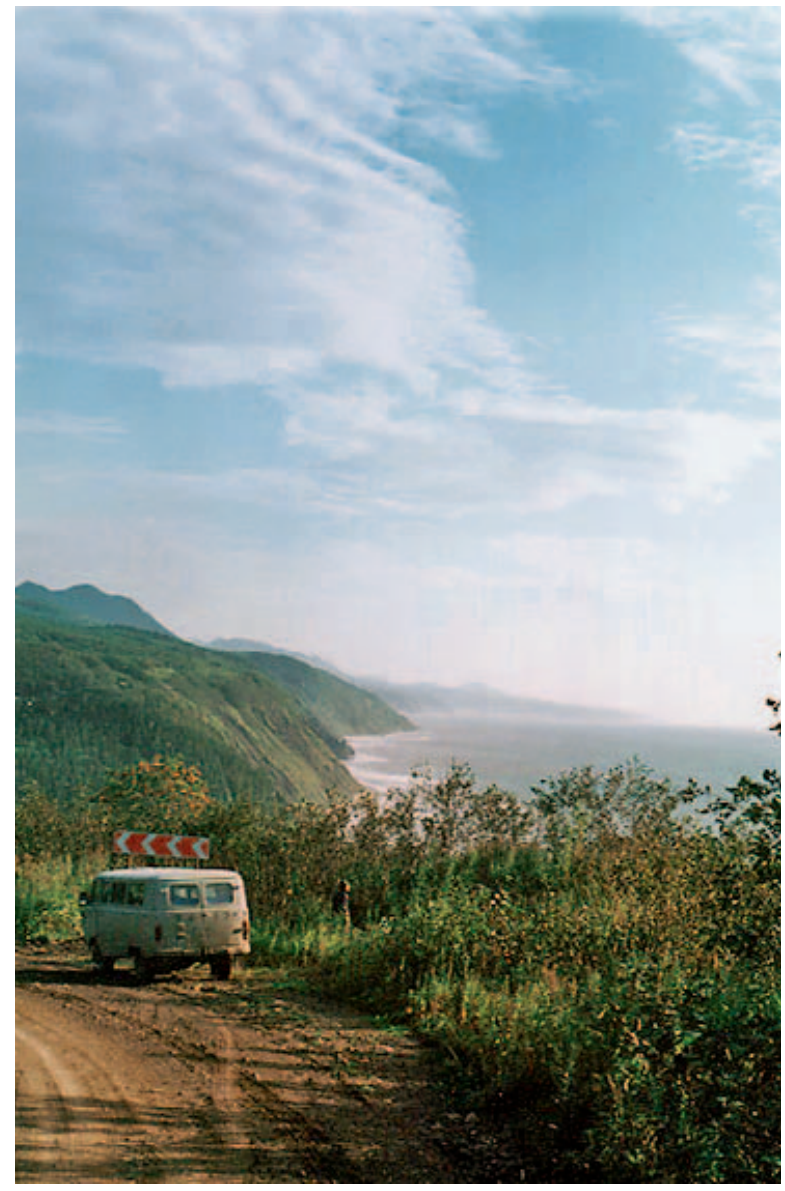

schiedenen Pflanzengesellschaften auf. Im Norden besiedelt sie Flussufer, in der Mitte kommt sie eher in Nadelwäldern auf Lichtungen und Brandstellen oder auf Wiesen entlang der Küste vor. Sie gehört auch zum Bestand des botanischen Gartens von Sachalin. Hier verträgt sie den Schatten unter Bäumen nur schlecht.

Die Blüten werden vor allem von Hummeln bestäubt und die Samen durch Vögel ausgebreitet. Die wichtigsten sind Wald- und Haselhühner. Der Strauch zeigt eine klare Anpassung an diese Fruchtfresser. Die Vögel halten sich vorwiegend auf dem Boden auf und dementsprechend hängen die Früchte an den niedrigen Sträuchern bis zum Boden herunter, während sie von oben kaum gesehen werden.

Seit in den 1980er Jahren auf Sachalin vermehrt Landhäuser gebaut und private Gärten angelegt werden, wurden (vom Festland) verschiedene Kultivare von $L$. caerulea eingeführt und erlangten Popularität. Mittlerweile sind auch über die regionale Agrarverwaltung verschiedene Kultivare zu beziehen. Wildentnahmen werden dagegen in den privaten Gärten kaum kultiviert.

\section{Lonicera tolmatchevii POJARK}

Der 1,8 m hohe Strauch hat vierkantige Triebe. Seine elliptischen bis eiförmigen Blätter sind $6-8 \mathrm{~cm}$ lang und $4-5 \mathrm{~cm}$ breit. Die Blüten werden in den Achseln der unteren vier Blattpaare eines Triebes gebildet. Ihre Tragblätter sind rundlich bis dreieckig-eiförmig, unverwachsen und grünlichrot. Die Farbe der 15-17 mm langen Blüten reicht von Gelb bis Grünlich-Gelb. Bisweilen sind sie purpurn überlaufen. Die kugeligen Früchte sind bis $9 \mathrm{~mm}$ breit, unverwachsen, schwarz und von bitterem Geschmack.

Diese Geißblatt-Art ist ein Endemit der Wälder entlang des Tym-Flusses im Zentrum Sachalins. Einzelne Sträucher bilden rasch große Klone und undurchdringliche Dickichte. Bei Überschwemmungen werden einzelne Teile weggerissen. An anderen Stellen entlang des Flusses können sie wieder Fuß fassen.

Abb. 1: Landschaft an der Westküste Sachalins. 
Der größte Teil des ursprünglichen Areals ging durch den Anstieg des Meeresspiegels zu Beginn des Holozäns verloren (Nedoluzhкo 1983). Vermutlich gedieh die Art früher entlang von Flüssen, die durch weite Ebenen verliefen, die heute vom Meer überschwemmt sind. Eine eng verwandte Art, Lonicera involucrata, konnte sich besser halten und drang bis nach Amerika (Rocky Mountains, Oregon, Utah) vor.

Der Tym-Fluss ist nur $65 \mathrm{~km}$ lang. An seinen Ufern sind bisher nur 12 Standorte der seltenen L. tolmatchevii bekannt. Diese verteilen sich auf ein Gebiet mit einer Fläche von 70 km² $^{2}$ Lonicera tolmatchevii bevorzugt sandige Böden mit guter Drainage, wird aber jährlich auch überschwemmt. Mit ihr vergesellschaftet sind Populus maximowiczii, Salix schwerinii und S. udensis. Lonicera tolmatchevii ist auf Sachalin eine bedrohte Art. Die Standorte sind durch Landwirtschaft, Straßenbau, Ölleitungen und Siedlungen stark bedroht. Es wäre sinnvoll, diese seltene Art in Kultur zu nehmen, um sie damit vor der Gefahr des Aussterbens zu bewahren.

Im botanischen Garten von Sachalin blüht und fruchtet $L$. tolmatchevii regelmäßig. Die Früchte reifen dort schon in der zweiten Junihälfte, früher als fast alle anderen Lonicera-Arten auf Sachalin. Am Wildstandort wurden bisher noch keine Sämlinge gefunden. Im botanischen Garten beträgt die Keimungsrate $90 \%$.

\section{Lonicera glehnii Fr. SCHMIDT}

Dieses Geißblatt ist nur schwach verzweigt und wird bis $2 \mathrm{~m}$ hoch. Seine abblätternde Borke hängt an den Stämmen herunter. Die Blätter des Strauches sind 6-8 cm lang. In den Achseln der oberen Blätter eines Zweiges bilden sich die Blüten. Ihre Krone ist 10-14 mm lang, zweilippig und grünlichgelb gefärbt mit einem Hauch von purpurn. Selten sind die Blüten leuchtend rosa gefärbt. Die beiden Beeren sind komplett miteinander verwachsen und bilden ein abgeflachtes, leuchtend rotes, kirschgroßes Synkarpium. Dieses hat bitteren Geschmack.

Abb. 2 (oben): Lonicera caerulea.

Abb. 3 (unten): Lonicera tolmatchevii.
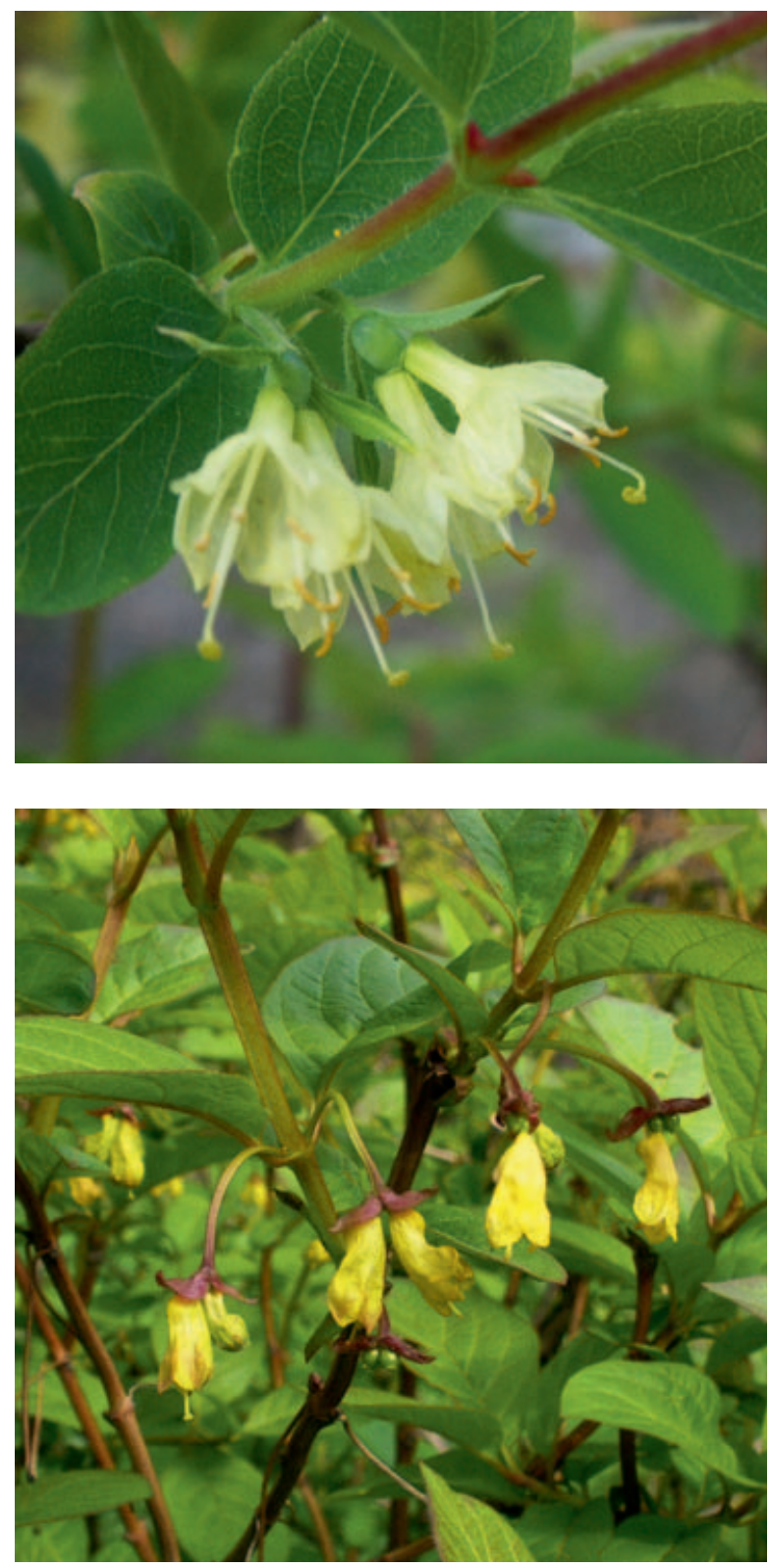

Lonicera glehnii ist im Süden und in der Mitte von Sachalin verbreitet. Es bevorzugt dunkle Nadelwälder und die unteren Bereiche der Betula-ermannii-Wälder. Es wächst meist entlang von Flüssen. In den Flusstälern gedeiht es dort am besten, wo es nicht ständig überschwemmt wird. Kräftige Frühjahrshochwasser übersteht es nur schlecht. Dagegen fühlt es sich sehr wohl in sekundären Birkenwäldern, die auf Kahlschlägen in Fichtenwäldern aufkommen. Im Gegensatz zur vorgenannten Art bildet $L$. glehnii keine Dickichte, da ihr die Fähigkeit zur vegetativen Vermehrung fehlt.

Vermutlich ist L. glehnii ein Relikt der alten Turgajskaya-Flora, die sich auf Sachalin in Zeiten 

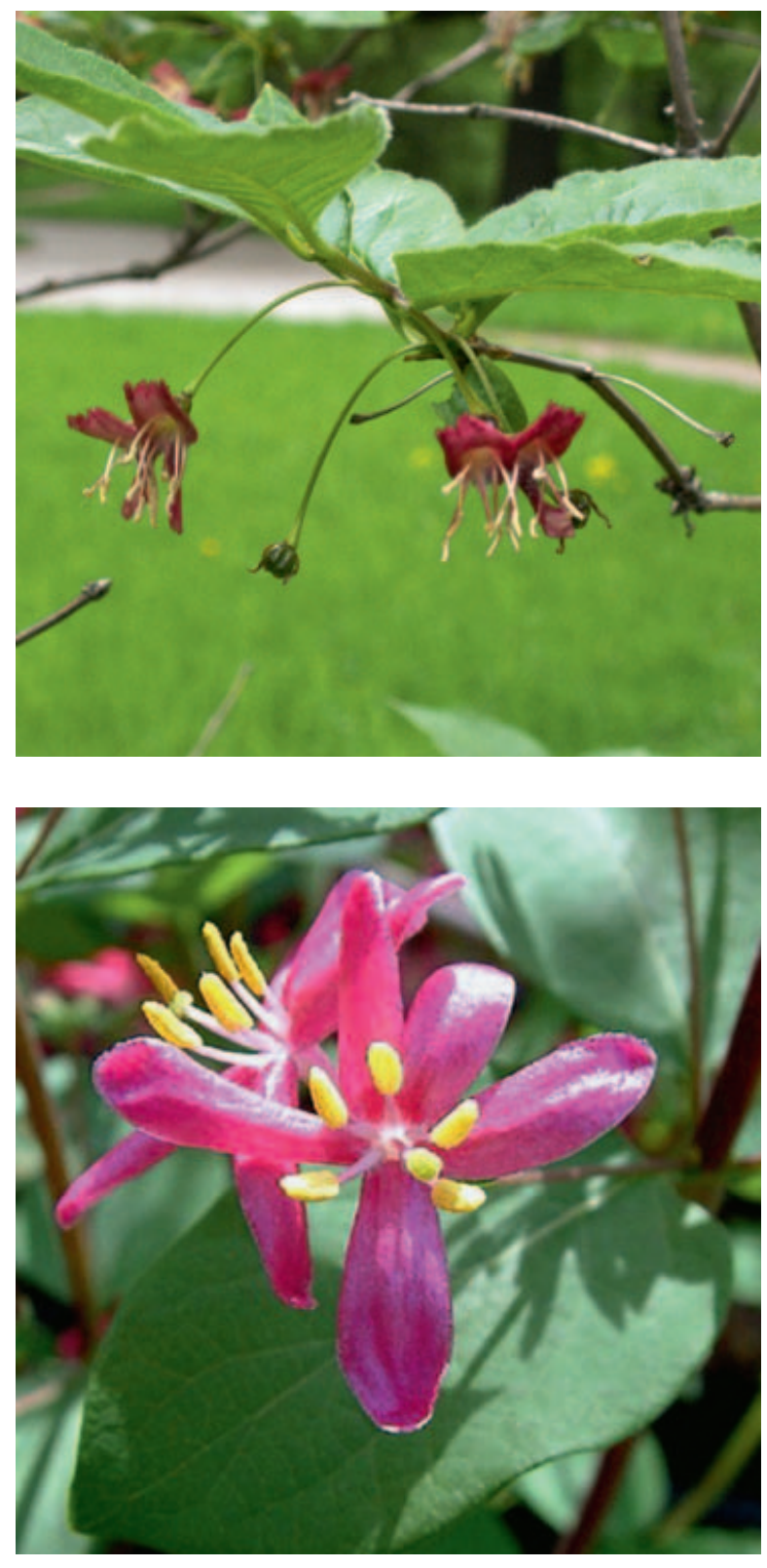

wärmerer Winter vor den Eiszeiten entwickelt hat. Auf Hokkaido, wo heute die Winter wärmer als auf Sachalin sind, kommt L. glehnii auch vor. Dort steigt sie in den Bergen nicht höher als auf 800 m (Hara 1983).

\section{Lonicera maximowiczii subsp. sachalinensis} (Fr. SCHMIDT) NedOLUZHKO

Die Blätter des bis 2,5 m hohen Strauches werden 5-6 cm lang. Die Tragblätter der Blüten sind pfriemförmig und nur $2 \mathrm{~mm}$ lang. Die purpurviolette Blütenkrone ist zweilippig und bis $14 \mathrm{~mm}$ lang. Nur wenige Minuten nach dem Verzehr der Früchte entsteht im Mund ein brennendes Gefühl.
Dieses Geißblatt kommt überwiegend im Süden Sachalins vor. Es wächst an ökologisch ganz verschiedenen Standorten. Zum einen ist es in Betula-ermannii-Wäldern über $800 \mathrm{~m}$ anzutreffen, andererseits gedeiht es in Lärchenwäldern in Tälern und Ebenen. Weitere Standorte sind Eichenwälder in Küstennähe. Im Winter werden ältere Zweige unter der schweren Schneelast zum Boden gedrückt, wo sie sich im Sommer bewurzeln. Dadurch bildet L. maximowiczii ausgedehnte Dickichte. Mit seinen leuchtend gelben Blättern ist der Strauch im Herbst besonders attraktiv.

\section{Lonicera chamissoi BUNGE}

Der nur $1 \mathrm{~m}$ hohe Strauch hat kleine, nur 3-4 cm lange Blätter. Die Blüten sind purpurviolett gefärbt und 9-12 mm lang. Die rundlichen, miteinander verwachsenen Früchte sind rot gefärbt mit wachsartigem Überzug und schmecken bitter. Es gibt kontroverse Meinungen darüber, ob die Früchte giftig sind oder nicht.

Lonicera chamissoi kommt auf ganz Sachalin vor und ist auch vom Russischen Fernen Osten bekannt. Auf Sachalin ist der Strauch sowohl in der subalpinen Stufe als auch im Flachland anzutreffen. Er kommt häufig auf Kahlschlägen, Brandstellen, kahlen Berggipfeln und entlang von Straßen vor. In den Bergen ist er meist in Lagen über $700 \mathrm{~m}$ zu finden. Unterhalb der Nadelwälder trifft man L. chamissoi in Lärchenwäldern und auf Meeresterrassen an. Diese Geißblatt-Art ist häufig mit Vaccinium axillare, $V$. hirtum, V. praestans, Sorbus sambucifolius, Pinus pumila, Acer ukurunduense und Ilex rugosa vergesellschaftet. An der Küste wächst sie z. B. zusammen mit Juniperus sibirica und Rosa rugosa.

Lonicera chamissoi wird im botanischen Garten von Sachalin kultiviert. Sie stellt geringe Ansprüche an den Boden und ist sehr wüchsig. In der Natur wächst sie dort am besten, wo die kleinen Sträucher im Winter von einer hohen

Abb. 4 (oben): Lonicera glehnii.

Abb. 5 (unten): Lonicera maximowiczii subsp. sachalinensis. 
Schneedecke vor Frostschäden geschützt sind (Nedoluzhko 1986). Jungpflanzen sind durch sporadisch auftretende Fröste im Sommer gefährdet. Eine Strategie, diese Gefahr zu minimieren, besteht in der frühen Frucht- und Samenreife. Die Samen können in der Mitte des Sommers keimen, wenn die Temperaturen besonders hoch sind.

Erstaunlicherweise sind enge Verwandte von L. chamissoi in deutlich wärmeren Gebieten verbreitet. So kommen L. subsessilis und L. barbinervis in Korea vor und L. nigra wächst in Bergwäldern Mitteleuropas.

\section{Lonicera chrysantha TURCZ. ex LEDEB.}

Mit $4 \mathrm{~m}$ Höhe gehört diese Art zu den größeren Loniceren. Im Allgemeinen hat der Strauch zwei unterschiedlich dicke Hauptstämme mit grauer Borke. Die Blätter sind 6-12 cm lang und $3-5 \mathrm{~cm}$ breit. Seine zweilippigen Blüten sind 14-19 mm lang, blassgelb, weiß oder grünlich gefärbt. Die runden, 5-8 mm großen Früchte sind unverwachsen und rot. Sie schmecken bitter und sind giftig. Die Art ist eng verwandt mit der in Mitteleuropa verbreiteten L. xylosteum, deren Früchte ebenfalls giftig sind. Auf Sachalin kommt L. chrysantha in Flusstälern und in den unteren Regionen der Berge vor. Bevorzugte Standorte befinden sich in sekundären Birkenwäldern und Gebüschen. Häufig ist L. chrysantha auch am Fuß von Flussterrassen in südlicher und westlicher Exposition anzutreffen. An der Küste kommt die Art auch im Grasland vor. Lonicera chrysantha besiedelt zwar meist gute Böden, gedeiht aber auch auf mageren Standorten.

Die Früchte reifen relativ spät im August und September. Die Samen keimen schnell und die Keimlinge wachsen rasch, so dass sich an den Jungpflanzen bis zum Winter zwei Blattpaare entwickeln können. Im Winter sind sie durch hohe Schneebedeckung gut geschützt. Neben Sambucus nigra ist $L$. chrysantha die einzige Art

Abb. 6 (oben): Lonicera chamissoi.

Abb. 7 (unten): Lonicera chrysantha.
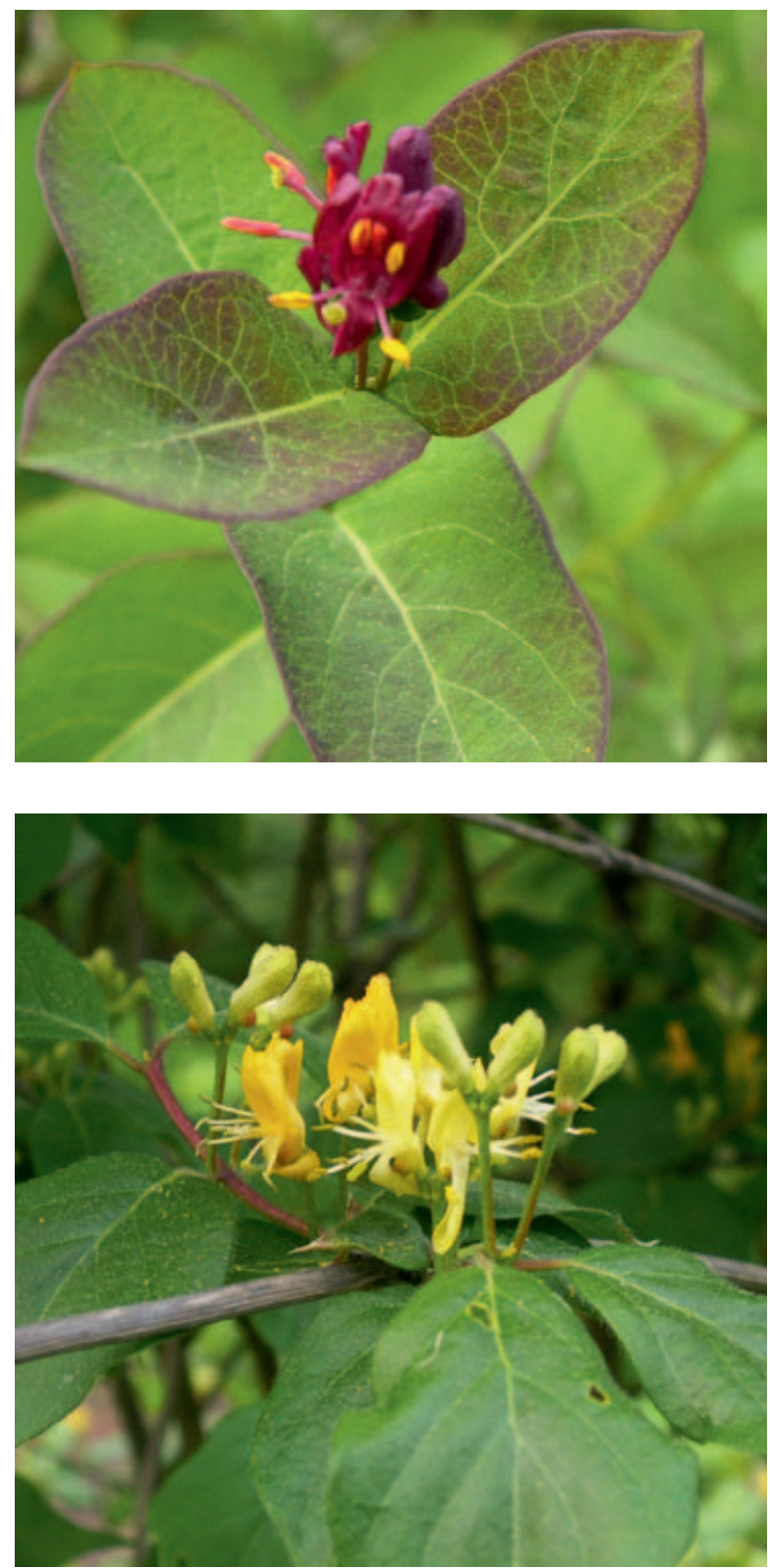

auf Sachalin, die deutlich unter Schneebruch leidet. Abgestorbene Äste werden in der folgenden Vegetationsperiode durch Neuaustriebe ersetzt.

Es wird vermutet, dass L. chrysantha die Insel vom Festland aus besiedelte. In Russland wird sie häufig kultiviert, ist sehr hart und an Bedingungen in den Städten angepasst.

\section{Schlussbetrachtung}

Die sechs Lonicera-Arten auf Sachalin nehmen eine unterschiedliche floristische Stellung ein. Lonicera caeruela ist ein häufiges boreales Florenelement, während $L$. tolmatchevii ein seltener Reliktendemit mit einem sehr kleinen Areal ist. 
Lonicera glehnii ist aus der Turgajskaya-Flora erhalten, die ein Refugium vor niedrigen Wintertemperaturen in den etwas geschützten dichten Nadelwäldern gefunden hat. Dagegen ist L. maximowiczii subsp. sachalinensis ein Florenelement des Raumes Sachalin-Hokkaido, das vor Anstieg des Meeresspiegels an Flüssen verbreitet war, die es heute nicht mehr gibt. Wohl eher ein Vertreter einer subtropisch verbreiteten Lonicera-Gruppe ist L. chamissoi. Diese Art kann sich in der subalpinen Höhenlage aufgrund der winterlichen, schützenden Schneedecke halten. Ein Element der Mandschurischen Flora, das in das Gebiet von Sachalin und Hokkaido einwandern konnte, ist Lonicera chrysantha.
Alle sechs Lonicera-Arten haben dekorative Früchte und sind Honigpflanzen, weshalb sie als Zierpflanzen zu empfehlen sind. Sie lassen sich vermutlich in Westeuropa gut kultivieren. In ihrer Heimat auf der Insel Sachalin sollten sie, und vor allem auch ihre Lebensräume, geschützt werden.

\section{Literatur}

Hara, H. 1983: A revision of Caprifoliaceae of Japan with reference to allied plants in other districts and the Adoxaceae. - Tokyo.

Nedoluzhro, V. A. 1983: Zhimolost Tolmacheva na

Sahaline. - Bul. Glav. Botan. Sada. 127: 29-34.

Nedoluzhко V. A. 1986: Sistematichesky i

geografichesky obzor zhimolostej severo. - Vladivostok.

\section{Gärtnerisch-botanische Literatur}

Horst Kretzschmar, Wolfgang Eccarius \& Helga DiETRICH

Die Orchideengattungen Anacampsis, Orchis, Neotinea. - Phylogenie, Taxonomie, Morphologie, Biologie, Verbreitung, Ökologie, Hybridisation.

EchinoMedia, Bürgel, 2007, 544 Seiten, zahlreiche farbige Abbildungen, gebunden, 89 Euro; ISBN 978-3-937107$11-0$.

Orchideen gehören zu den artenreichsten Pflanzenfamilien. Vertreter dieser Pflanzengruppe, ob es nun üppige, großblütige tropische Orchideen oder die etwas unscheinbareren europäischen Arten sind, finden viele Freunde. Nicht umsonst widmete sich erst kürzlich eine große Informationsausstellung des Palmengartens der Biologie und Kulturgeschichte der Orchideen.

Es ist nicht immer leicht, die einzelnen Arten oder gar Hybriden auseinander zu halten. Mit Hilfe dieses Buches soll Abhilfe geschaffen, gleichzeitig die Vielfalt demonstriert werden. In einem einführenden Kapitel wird auf die Geschichte der Erforschung der Orchideen, besonders der in diesem Buch behandelten Gattungen, eingegangen. Dabei werden auch historische Abbildungen aus dem Kräuterbuch von Leonhart Fuchs gezeigt. Der Leser findet hier zudem Abbildungen wichtiger Orchideenforscher wie z. B. von John Lindley, Heinrich Gustav Reichenbach oder Rudolf SCHLECHTER.
Es werden neuere Gattungs- und Artkonzepte und dazu passende Kladogramme vorgestellt. Das Buch enthält einen Gattungs-Bestimmungsschlüssel für Anacampsis, Neotinea und Orchis, zudem sind die einzelnen Arten der jeweiligen Gattungen aufgeschlüsselt. Den größten Teil des Buches nehmen die einzelnen Artbeschreibungen ein. Dazu gehören brillante Farbfotos der gesamten Pflanze, Blütendetails und Standortsaufnahmen. Diverse Hybriden zwischen Anacampsis und Serapias werden übersichtlich auf einer Farbtafel mit Zeichnungen nebeneinander gestellt. Ferner gibt es Angaben zur Synonomie, Morphologie, Biologie, Zytologie, Ökologie, Variabilität, sowie zum Bestand und zur Gefährdung. Die Verbreitung wird anhand eines kurzen Textes und einer Karte veranschaulicht. Für denjenigen, der sich vertieft mit der Orchideentaxonomie beschäftigt, ist es hilfreich, hier Angaben zur Erstbeschreibung sowie zu Typusbelegen zu finden.

Das vorliegende Buch birgt nicht nur eine Menge Fleißarbeit und großen Zeitaufwand bei dem Zusammentragen der Merkmale sowie der Fotos. Für den Freund europäischer Orchideen ist es eine gute Bestimmungshilfe, vor allem auch der schwierigen Hybriden. Es bietet vor allem aber auch dem Orchideenspezialisten eine Menge wichtiger Informationen. Die Kladogramme dürften vor allem die Fortgeschrittenen interessieren. Der noch weiter Interessierte findet am Ende des Buches eine umfangreiche Literaturliste.

Der Preis des Buches ist zwar recht hoch, ist jedoch bei der hervorragenden Ausstattung gerechtfertigt.

Hilke Steinecke 